\title{
New Directions for Therapeutics in ADHD
}

\author{
Julie B. Schweitzer • Keith McBurnett
}

Published online: 2 August 2012

(C) The American Society for Experimental NeuroTherapeutics, Inc. 2012

The number of publications on attention deficit hyperactivity disorder (ADHD) has increased sharply over the last 10 years, with more than 700 likely for 2012. Consequently, it is difficult to stay apprised of this literature. This issue of Neurotherapeutics includes 12 comprehensive reviews of topics related to ADHD.

ADHD is the most common childhood behavioral disorder and one of the more common adult psychiatric disorders. Traditional therapeutics for ADHD include behavioral therapy and pharmacologic intervention. These approaches have been available for decades; however, therapies have evolved and expanded with better understanding of the disorder. This includes a better understanding of the role of dopamine and norepinephrine and how this new knowledge can guide the development of novel pharmacological agents. Better recognition of subpopulations of the disorder, the presence of co-morbidity (e.g., sleep disturbance and autism spectrum disorders), and need to address the disorder throughout the life span have helped refine the diagnostic process. The search for improved treatments for ADHD continues, as a significant portion of the ADHD population does not respond, or responds sub-optimally, to currently available therapy. Concerns about drug side effects have fostered an interest in identifying new and alternative treatment options.

\footnotetext{
J. B. Schweitzer $(\bowtie)$

Department of Psychiatry and Behavioral Sciences and MIND Institute, University of California Davis School of Medicine, 2825 50th St,

Sacramento, CA 95817, USA

e-mail: julie.schweitzer@ucdmc.ucdavis.edu

K. McBurnett

Department of Psychiatry, University of California-San Francisco,

San Francisco, CA, USA
}

The current issue reviews new approaches that range from preventative interventions that minimize the development of the disorder to the potential for treatment based on electrical brain response. Similarly, the role of environmental impact, such as food and food dyes, on behavior associated with ADHD, is addressed. Also critically reviewed are current conceptualizations of the disorder and potential new targets for treatment. The latter articles include discussion of prevalence of the disorder and subtypes, the role of variability in response times in ADHD, cognitive training approaches for targeting reward-related and cognitive impairments, among others.

Willcutt [1] (this issue) report the largest meta-analysis to date on the prevalence of ADHD, along with related analyses of a large population-based sample. Prevalence varied according to diagnostic methodology, but using a bestestimate procedure and strict DSM-IV diagnostic criteria, the overall incidence of ADHD was estimated to be $5.9 \%$, and similar across countries and regions. Tests of changes proposed for diagnosing ADHD in DSM-V found little effect for relaxing the age of onset criterion or allowing co-morbid psychotic or pervasive developmental disorders. In contrast to the wealth of data on children, much less is known about ADHD in adults. Green and Rabiner [2] (this issue) contribute a qualitative discussion of the small volume of literature available on ADHD in college students. Among the difficulties with diagnosing and treating ADHD in college students his group noted are the high rates of malingering in order to obtain prescription medication, and the frequency of diversion and misuse of medication.

Children with ADHD are increasingly recognized as "consistently variable" in their performance. Parents and teachers have noted for years that children seem to vary considerably day to day in their attention, participation, and ability to follow directions. This variability can be frustrating to both the child and caregivers. It is only within the past decade that variability in ADHD has been studied systematically. Much of this research relates to intra-individual 
variability in reaction time (RT) on computerized tasks. Heightened RT variability is now considered one of the most common findings in ADHD.

Tamm et al. [3], review the literature on variability in RT and its specificity to ADHD, and discuss the clinical and neural correlates of RT variability. The authors emphasize the importance of carefully selecting the measures and context in which RT variability is assessed. The effect of pharmacologic and nonpharmacologic interventions on RT variability is also reviewed.

Conventional clinical wisdom is that artificial food colors (AFCs) are not a major cause of ADHD. In the included article by Arnold et al. [4] (this issue), the group agrees with this assessment, but argue in a detailed and nuanced review that (a) on a population basis, there is evidence of a small but significant deleterious effect of AFCs on children's behavior; (b) consumption of AFCs has been steadily increasing, particularly in the most recent decade; and (c) the aggregate effects of AFCs on entire classrooms may be underappreciated. They recommend that AFCs be studied more thoroughly as a public health problem.

Halperin et al. [5, this issue] also present a model and strategies for secondary prevention interventions for ADHD and early intervention in the disorder using nonpharmacological interventions [5] (this issue). Prevention of ADHD represents a novel paradigm shift just recently articulated by Halperin and colleagues [6]. The authors review evidence - targeting behaviors associated with ADHD during the pre-school years and suggest that these interventions are more likely to have long-lasting positive effects if they are implemented during this critical period of brain development when there is greater neuroplasticity. Their model is novel in its attempt to target executive functioning behaviors, rather than earlier intervention attempts that used more traditional behavior therapy approaches. They propose the use of environmental enrichment and physical exercise to enhance neurocognitive development and achieve their goals. Another obvious advantage of the approach proposed by Halperin and his colleagues is that these strategies are likely to strengthen positive behaviors in children with or without ADHD and across severity levels; thus, these strategies could be implemented on a wide scale.

Tripp and Wickens [7], this issue, update a theoretical model of ADHD, the dopamine transfer deficit (DTD) hypothesis. A central tenant is that associative learning involves the transfer of phasic dopamine signals from established positive reinforcers such as praise or attention to earlier cues that predict reinforcers, leading to long-term potentiation and strengthening of corticostriatal connections. This transfer is inefficient in cases of ADHD. The DTD hypothesis predicts that drugs that effectively treat ADHD may do so by acting at the dopamine transporter and thus compensating for a deficit in dopamine transfer, rather than reversing the primary deficit by acting on the circuitry that controls the dopamine neurons. The complementary review from Minzenberg [8] (this issue) discusses the broader view of neurochemistry and therapeutics, including catecholaminergic mechanisms at the cellular level, changes at the regional level from imaging studies, and performance changes at the behavioral level.

The relationship between sleep and ADHD is complex. Stein et al. [9], (this issue), review the evidence for the type of sleep problems present in children and adults with ADHD. They also discuss the effects of medication to treat ADHD on sleep. Exploring baseline sleep issues and treatment-related issues are crucial to the optimal functioning for an individual with ADHD. Issues related to interand intra-variability within and between patients with ADHD are also important, as the effects of medication on sleep can vary greatly within an individual from day-to-day, as well as among individuals with the diagnosis. Stein and colleagues present suggestions for clinical providers, and underscore the need for increased research into this challenging area.

We include an article reviewing the clinical utility of EEG in diagnosing and treating ADHD, Loo and Makeig [10], (this issue). They cite evidence for considering increased frontocentral theta band activity and increased theta-to-beta ratio as markers for ADHD, but they argue that methodological issues raise questions regarding the current utility of EEG to identify ADHD. In their assessment of EEG-based neurofeedback (NF) to treat ADHD, they suggest that EEG approaches may have some utility as adjunctive treatments (but not as monotherapy) in some individuals with ADHD. A complementary review from Moriyama et al. [11] (this issue) focuses on the treatment outcome literature. They conclude that NF may be an effective alternative treatment for ADHD, although more data are available from studies that used it as an adjunctive treatment. Both reviews agree that further research using better and more advanced methodologies is needed.

Strategies to target executive functioning and cognitive control impairments with older children, adolescents, and adults with ADHD is addressed by Rutledge et al. [12] in their article (this issue). They review the use of the dual pathway theory of ADHD [13] and its similarity to the dual systems model of self-control [14] and how the two models can be integrated and serve as a framework to target cognitive and reward-related impairments in ADHD. The article also posits that the developmental stage is an important consideration for implementing cognitive training strategies in ADHD. The authors suggest that adolescence may be a particularly important period for cognitive training as early adolescence is a period of brain development in regions (e.g., prefrontal and parietal cortices) associated with the development of cognitive control. Thus, by intervening at this critical stage in development, effective cognitive training could lead to better 
regulation of impairments found in ADHD. They also discuss research from human operant and behavioral economic approaches and how these can serve as resources for targeting reward-related impairments in ADHD.

The presence of significant ADHD symptoms in persons with autism spectrum disorders (ASD) is well recognized, yet only recently has there been sufficient information to guide treatment of the symptoms. Similarly, there exists a sizeable portion of individuals with diagnosable ADHD who have sub-threshold ASD symptoms; some may have presented with more severe ASD symptoms when younger and received early intervention. They may retain some difficulties with social functioning, but their significant executive functioning deficits, including classic problems associated with ADHD (i.e., inattention and/or hyperactivity and impulsivity) interfere with both school functioning and personal relationships. The need for effective interventions in these populations is just starting to be addressed. Davis and Kollins [15] review pharmacological and psychosocial interventions, (this issue), and suggest future research areas based on current gaps in the knowledge regarding the co-occurrence of ADHD and ASD symptoms.

Together, these reviews provide a timely and wideranging update of ADHD diagnosis and treatment.

Required Author Forms Disclosure forms provided by the authors are available with the online version of this article.

\section{References}

1. Willcutt EG. The prevalence of DSM-IV attention-deficit/hyperactivity disorder: A meta analytic review. Neurotherapeutics. 2012; doi:10.1007/s13311-012-0135-8.
2. Green AL, Rabiner DL. What do we really know about ADHD in college students? Neurotherapeutics. 2012; doi:10.1007/s13311012-0127-8.

3. Tamm L, Narad ME, Antonini TN, O’Brien KM, Hawk LW, Epstein JN. Reaction time variability in ADHD: A review. Neurotherapeutics. doi:10.1007/s13311-012-0138-5.

4. Arnold LE, Lofthouse N, Hurt E. Artificial food colors and attention-deficit/hyperactivity symptoms: conclusions to dye for. Neurotherapeutics. 2012; doi:10.1007/s13311-012-0133-x.

5. Halperin JM, Bédard AV, Curchack-Lichtin JT. Preventive interventions for ADHD: a neurodevelopmental perspective. Neurotherapeutics. 2012; doi:10.1007/s13311-012-0123-z.

6. Sonuga-Barke EJ, Halperin JM. Developmental phenotypes and causal pathways in attention deficit/hyperactivity disorder: potential targets for early intervention? J Child Psychol Psychiatry. 2010 Apr;51(4):368-89.

7. Tripp G, Wickens J. Reinforcement, dopamine and rodent models in drug development for ADHD. Neurotherapeutics. 2012; doi:10.1007/s13311-012-0132-y.

8. Minzenberg MJ. Pharmacotherapy for attention-deficit/hyperactivity disorder: from cells to circuits. Neurotherapeutics. 2012; doi:10.1007/s13311-012-0128-7.

9. Stein MA, Weiss M, Hlavaty L. ADHD treatments, sleep, and sleep problems: complex associations. Neurotherapeutics. 2012; doi:10.1007/s13311-012-0130-0.

10. Loo SK, Makeig S. Clinical usefulness of EEG in attention-deficit/ hyperactivity disorder: a research update. Neurotherapeutics. 2012; doi:10.1007/s13311-012-0131-z.

11. Moriyama TS, Polanczy GV, Caye A, Banaschewski T, Brandeis D, Rohde LA. Evidence based information on the clinical use of neurofeedback for ADHD. Neurotherapeutics. 2012; doi:10.1007/ s13311-012-0136-7.

12. Rutledge KJ, van den Bos W, McClure SM, Schweitzer JB. Training cognition in ADHD: current findings, borrowed concepts and future directions. Neurotherapeutics. 2012; doi:10.1007/s13311012-0134-9.

13. Sonuga-Barke EJ. Psychological heterogeneity in $\mathrm{AD} / \mathrm{HD}$ - a dual pathway model of behaviour and cognition. Behav Brain Res. 2002;130(1-2):29-36. Epub 2002/02/28.

14. McClure SM, Laibson DI, Loewenstein G, Cohen JD. Separate neural systems value immediate and delayed monetary rewards. Science. 2004;306(5695):503-7. Epub 2004/10/16.

15. Davis NO, Kollins SH. Treatment for co-occurring attention deficit/hyperactivity disorder and autism spectrum disorder. Neurotherapeutics. 2012; doi:10.1007/s13311-012-0126-9. 